\title{
Simulations and experiments showing the origin of multiwavelength mode locking in femtosecond, Yb-fiber lasers
}

\author{
James S. Feehan, ${ }^{1, *}$ F. Ömer Ilday, ${ }^{2,3}$ William S. Brocklesby, ${ }^{1}$ and Jonathan H. V. Price ${ }^{1}$ \\ 'Optoelectronics Research Centre, University of Southampton, Hampshire SO17 1BJ, UK \\ ${ }^{2}$ Department of Physics, Bilkent University, 06800 Ankara, Turkey \\ ${ }^{3}$ Department of Electrical and Electronics Engineering, Bilkent University, 06800 Ankara, Turkey \\ ${ }^{*}$ Corresponding author: jamesfeehan56@googlemail.com
}

Received 26 April 2016; revised 26 June 2016; accepted 27 June 2016; posted 28 June 2016 (Doc. ID 264006); published 15 July 2016

\begin{abstract}
A stable and self-starting femtosecond breathing-pulse $\mathrm{Yb}$-fiber oscillator is reported, mode-locked using the nonlinear polarization evolution mechanism. A bifurcation between two distinct modes of operation is demonstrated experimentally, producing pulses with a single central wavelength in one state, or following adjustment of the intracavity waveplates, the emission of pulses with three distinct central wavelengths. The maximum bandwidth was $72 \mathrm{~nm}$ at the $-10 \mathrm{~dB}$ level, and the pulses were compressible externally to $70 \mathrm{fs}$ with energies of $0.75 \mathrm{~nJ}$. The multiwavelength pulses reported here are significantly shorter than the picosecond pulses previously observed from similar mode-locked multiwavelength sources. Vector simulations based on the nonlinear Schrödinger equation show that the multiwavelength behavior is produced by overdriving the nonlinear polarization evolution-based saturable absorber at the peak of the pulse, leading to transmission of the two wings of the strongly chirped pulse. This new insight shows clearly that the three pulses output in the multiwavelength state are coherent. The agreement between simulation and experimental data shows nonlinear polarization evolutionbased mode-locked fiber lasers are a suitable platform for studying the nonlinear dynamics underlying the bifurcation of the output. () 2016 Optical Society of America
\end{abstract}

OCIS codes: (320.7090) Ultrafast lasers; (140.4050) Mode-locked lasers; (060.2320) Fiber optics amplifiers and oscillators; (190.7110) Ultrafast nonlinear optics; (190.1450) Bistability.

\section{INTRODUCTION}

Since the discovery of first soliton-based mode locking and then breathing-pulse mode locking in fiber oscillators, there has been significant progress in the understanding of how to optimize the nonlinear cavity dynamics for either enhanced stability of operation or to produce higher energy pulses [1]. As well as traditional mechanisms based on nonlinear polarization evolution (NPE) arising from the Kerr effect in the fibers [2], various external materials have been harnessed to create the required fast switches in such cavities, such as semiconductor saturable absorber mirrors (SESAMs) [3,4] or, more recently, saturable absorbers based on two-dimensional materials such as $\mathrm{MoS}_{2}$ and graphene [5-10]). While some recent research has focused on polarization-maintaining (PM) fiber cavities [11], a large number of cavities have continued to use non-PM fibers and with NPE as a fast saturable absorber, either in combination with a SESAM or, as here, acting in tandem with a spectral filter.

With wide-ranging applications for multiwavelength laser sources in areas such as time-resolved spectroscopy, terahertz generation, and optical sensing, compact and stable modelocked fiber oscillators producing ultrafast optical pulses with different central wavelengths have seen considerable interest. To date, such sources have generally been reported in a phenomenological manner, with strong focus on experimental observation alone and little research into the cavity dynamics, slowing developmental progress. During the development of a $1045 \mathrm{~nm}$ laser for applications research, we observed an unusually broadband multiwavelength mode-locking state in a breathing-pulse $\mathrm{Yb}$-fiber cavity. This is described here both experimentally and using fully vectorial simulations which, for the first time, demonstrate what are, to the best of our knowledge, the shortest multiwavelength pulses achieved from a mode-locked cavity to date, as well as a full description of the underlying processes and start-up dynamics responsible for the multiwavelength operation of the cavity. This paper therefore represents the first detailed report of multiwavelength operation created by overdriven nonlinear evolution of the intracavity polarization state since breathing pulse femtosecond $\mathrm{Yb}$-fiber 
lasers were first developed more than two decades ago. Given the robust nature of the laser, this technique may prove useful for the above applications, which specifically require multiwavelength pulse trains.

Suggestions for the origin of multiwavelength output from NPE mode-locked cavities have not in general been underpinned by numerical modeling that would conclusively identify the cause, and the reports that are most similar in terms of pulse evolution to the Yb-fiber cavity studied here were based on Er-fiber lasers, which have different dispersion and gain characteristics. In addition to the increased number of cavity components normally used, the pulses produced are generally restricted to the $\sim 1$ ps range. Specifically, the output of multiwavelength pulse trains from an ultrafast Er-fiber oscillator was reported by Luo et al. [12], using a linear tuneable Lyot filter to form up to four frequency bands near $1580 \mathrm{~nm}$ with bandwidths of $\sim 0.75 \mathrm{~nm}$. The spacing of the wavelength bands was determined directly by the filter. An alternative method of obtaining multiwavelength output has been the inclusion of wavelength-selective components, such as fiber Bragg gratings [13]. Another report of multiwavelength output from a modelocked fiber laser attributed the behavior to the action of the fiber birefringence. The setup used dissipative solitons combined with a nonlinear optical loop mirror in a figure-of-eight cavity [14]. The pulses had bandwidths of around $10 \mathrm{~nm}$ and central wavelengths of $1572 \mathrm{~nm}$ and $1587 \mathrm{~nm}$. However, no attempt was made to externally compress the output pulses, so the degree of pulse coherence was not demonstrated.

The way that NPE or a nonlinear loop mirror can create multiple stable points of operation is illustrated in the first row of Fig. 1. The plot shows the transmission profile of the NPE fast saturable absorber as a function of intensity, and how this creates the multipeak transmission profile for a chirped pulse as the peak power is increased and eventually overdrives the NPE. The pulse peak can then be positioned at a local minimum in the transmission profile at intensity $I_{2}$, causing it to be rejected from the cavity, whereas the leading and trailing edges with intensity equal to $I_{1}$ experience maximum transmission so that two wavelengths are output at any subsequent tap coupler. This contrasts with the more generally observed case in which the peak of the chirped pulse would be aligned with the maximum in transmission at intensity $I_{1}$. To date, attention has been focused on the area near the first transmission maximum, which can lead to low-energy multipulsing, but wherein the pulses created have had similar wavelength characteristics, or high-energy single-pulse operation [15]. The mechanism of this pulse breakup in the time domain has been thoroughly investigated using simulations [16]. As we argue here, moving to a higher intensity point of operation underlies the bifurcation to multiple-wavelength operation when the pulse circulating in the cavity is chirped. Hence, extending the modeling to incorporate the NPE with full vector simulations provides a good foundation for studying how multiwavelength operation can be achieved.

In this paper, a femtosecond breathing-pulse $\mathrm{Yb}$-fiber ring laser, mode-locked using a saturable absorber based on NPE [17] is shown to exhibit multiwavelength operation that is purely due to variations of the circulated polarization state.

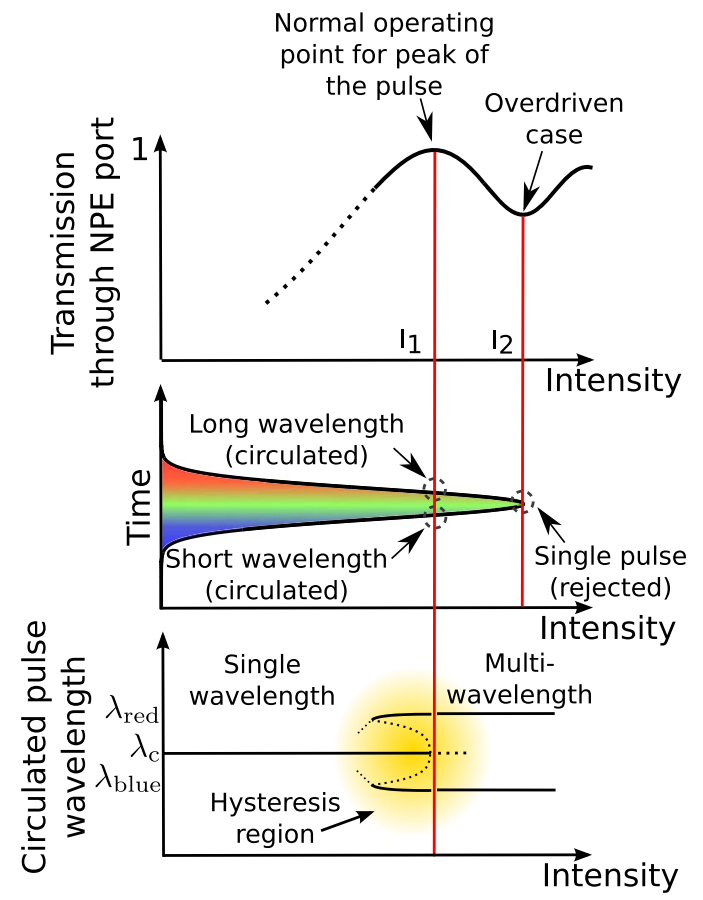

Fig. 1. Schematic of the multiwavelength operating principle. Upper plot: transmission through the NPE saturable absorber as a function of intensity. Middle plot: Gaussian pulse, with color map indicating chirp. The peak of the pulse at intensity $I_{2}$ sees a local minimum in transmission and is rejected from the cavity, whereas the points on the leading and trailing edge see a maximum in transmission, so remain in the cavity. Lower plot: qualitative depiction of the bifurcation between single-wavelength and multiwavelength operation of the laser.

By adjusting the intracavity waveplates, it was possible to switch from a single-wavelength mode of operation to one which produced output pulses with three distinct central wavelengths. The highest output pulse energy for the multiwavelength mode of operation was $0.75 \mathrm{~nJ}$, and this pulse was compressible to $70 \mathrm{fs}$ duration. The shortest pulse duration was $47 \mathrm{fs}$, with a pulse energy of $\sim 90 \mathrm{pJ}$. Simulations of the cavity dynamics based on the vector nonlinear Schrödinger equation are included and extend the work presented in Ref. [16] to show that this multiwavelength mode can be induced by variations in the ellipticity of the circulated polarization state and an overdrive of the NPE. Then, a mapping from the time domain to wavelength caused by the strong pulse chirp allows selection of multiple wavelengths at the saturable absorber. The changing strength of the nonlinear evolution enables the bifurcation of the single-wavelength state into the multiwavelength output. By focusing the simulations on the overdriven NPE switch, the cavity dispersion, and the resulting wavelength-varying polarization dynamics, we show that the multiwavelength operation arises directly from the combination of a temporally varying polarization state and chirped spectrum that are present at the polarization-based output switch.

The mechanism by which the NPE switch selects two separate wavelength bands and the associated subcritical pitchfork bifurcation and the hysteresis it leads to are illustrated in the 
second and third rows of Fig. 1. The strong chirp leads to the pulse wings passing through the overdriven NPE switch with separate short and long wavelength bands, while the center of the pulse is rejected and switched out of the cavity. In future, it may be possible to use NPE-based mode-locked fiber lasers for the study of the nonlinear dynamics of the bifurcation. Depending on the interplay of self-phase-modulation and Raman scattering, it may also be possible to observe further branching into four output wavelengths. Such links between mode-locked laser analysis and nonlinear dynamics have proven valuable in the study of rogue waves and turbulence [18].

The paper is structured as follows: the experimental cavity design and performance are described in Section 2. The simulations are detailed in Section 3. Then a brief discussion and conclusion presented in Section 4 provide comments on similarities and differences between simulations and the experiment, and their relation to other reports in the literature.

\section{EXPERIMENTAL LASER CHARACTERISTICS}

\section{A. Cavity Design}

The breathing-pulse ring cavity design was chosen, as this configuration generally produces pulses with higher output energies than a quasi-soliton laser [2], and because it often demonstrates low noise operation [19], which was required for our applications work. Most $\mathrm{Yb}$-fiber mode-locked cavities run at the secondary peak of the $\mathrm{Yb}$-fiber emission cross-section spectrum near $1030 \mathrm{~nm}$ to maximize the gain per unit length and hence, minimize the length of active fiber. Using fibers with high doping concentrations then enables fairly effective separation of gain and nonlinear effects, enabling higher output pulse energies in the $10 \mathrm{~nJ}$ region to be obtained routinely [20]. However, the cavity reported here had a target central wavelength of $1045 \mathrm{~nm}$, and thus used a longer length of gain fiber than usual. As a consequence, there was evidence of nonlinearity acting strongly at sub-nJ pulse energies. Although the cavity supported both single- and multiwavelength operation, only the multiwavelength results are presented, as this is the focus of the new findings reported here.

The ring cavity is shown in Fig. 2. A grating-based dispersive delay line (DDL) compensated for the fiber dispersion. Spectral filtering was applied using an iris in the DDL, which blocked wavelengths below $980 \mathrm{~nm}$ and above $1064 \mathrm{~nm}$. An additional filtering element was the fused fiber $980 \mathrm{~nm}: 1030 \mathrm{~nm}$ WDM pump coupler that had a $21 \mathrm{~dB}$ loss at $1082 \mathrm{~nm}$, as shown by the plot in Fig. 3. The spectral filtering formed an additional pulse-narrowing mechanism, which improves the self-starting cavity behavior, increases the nonlinearity of the pulse evolution and, in part, facilitates the creation of the broad output spectrum [21]. A $2.65 \mathrm{~m}$ length of HI1060 [Corning, Inc. (labeled SMF in the schematic)] and the WDM pigtails (OFS 980 fiber) provided normal dispersion. The $1.3 \mathrm{~m}$ length of $\mathrm{Yb}$-doped aluminosilicate fiber (2300 ppm) was pumped using a $976 \mathrm{~nm}$ diode with average power of $200 \mathrm{~mW}$ (measured after the WDM).

A $45 \mathrm{~cm}$ length of HI1060 was spliced onto the $\mathrm{Yb}$-fiber output to increase the nonlinearity. The cavity round-trip time was $\sim 25 \mathrm{~ns}(\sim 40 \mathrm{MHz}$ repetition rate) as measured from the radio frequency $(\mathrm{RF})$ spectrum. In the free-space section of the

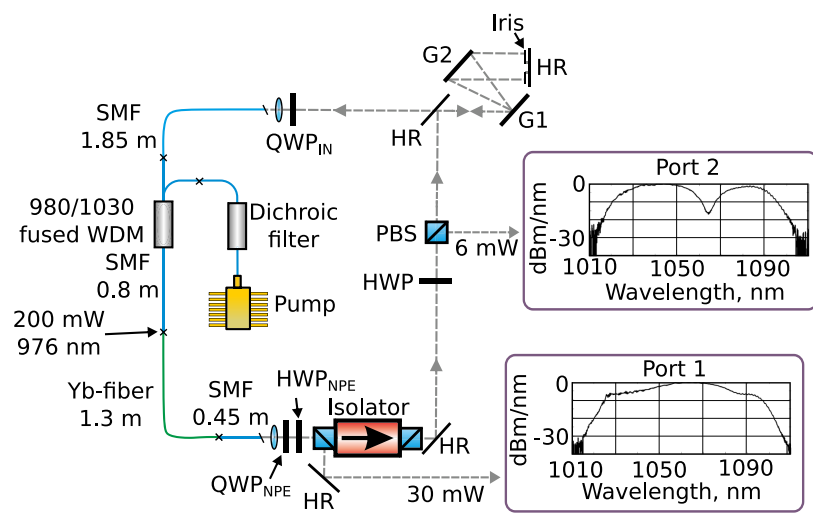

Fig. 2. Schematic of the mode-locked Yb-fiber oscillator. Insets show the spectra from the two output ports in multiwavelength operation. Port 2 shows a spectral minimum, positioned at the $1064 \mathrm{~nm}$ central wavelength of the output from port 1. (Abbreviations defined in the text.)

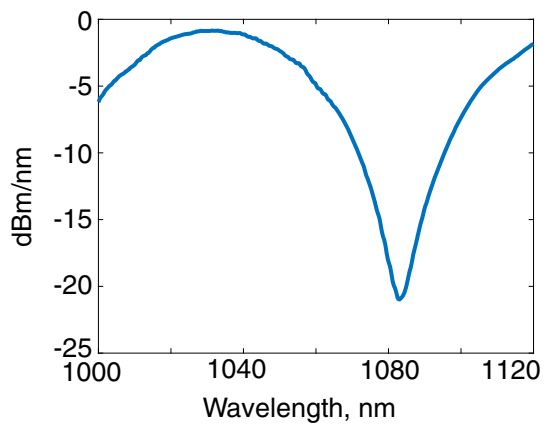

Fig. 3. Intracavity spectral filtering due to the WDM. There is a $21 \mathrm{~dB}$ loss at $\sim 1082 \mathrm{~nm}$.

cavity, a Faraday isolator ensured unidirectional operation and a quarter-waveplate (QWP), half-waveplate (HWP) and polarizing beam splitter (PBS) at the isolator input formed the NPE switch. The NPE rejected output is called port 1 . An additional HWP and PBS formed a variable output coupler, called port 2 .

The dispersion and nonlinearity of the cavity fibers were calculated using the manufacturer-specified core size and NA, and the standard parameters for silica glass [22]. Taking a typical fiber group velocity dispersion (GVD) of $\beta_{2}=2.3 \times$ $10^{4} \mathrm{fs}^{2} / \mathrm{m}$ and total length of $4.4 \mathrm{~m}$, the group delay dispersion (GDD) is $10.1 \times 10^{4} \mathrm{fs}^{2}$. The grating-based DDL (600 lines/ $\mathrm{mm}, \mathrm{G} 1$ and G2 in the diagram) provided GDD of approximately $-13 \times 10^{4} \mathrm{fs}^{2}$ (chosen as it allowed for a combination of reliable self-starting and good stability). Hence the cavity dispersion is $-2.9 \times 10^{4} \mathrm{fs}^{2}$ (i.e., slightly anomalous).

\section{B. Performance}

The laser demonstrated robust, self-starting operation with a single temporally isolated but multiwavelength pulse circulating in the cavity. The output pulse energies were $0.75 \mathrm{~nJ}$ and $0.15 \mathrm{~nJ}$ (powers of $30 \mathrm{~mW}$ and $6 \mathrm{~mW}$ ) from ports 1 and 2, respectively. The pulse duration measured directly from port 1 was $\sim 3$ ps as inferred from autocorrelation data, assuming a 
Gaussian pulse shape. At this point, the pulse has a positive linear chirp and is over 40 times the transform-limited duration. Port 1 outputs the central, highest peak power portion of the pulse that is rejected by the NPE switch. Port 2 is simply a power tap, so it outputs a fraction of the remaining parts of the pulse (i.e., the leading and trailing edge). There are no strongly dispersive elements between ports 1 and 2 , so the output chirp from both ports is similar, and hence, the duration and chirped pulse shape at port 2 can be inferred from a comparison of the output spectra shown in the inset in Fig. 2. The port 2 output coupling ratio was $\sim 40 \%$. Increasing the circulated pulse energy (e.g., by either increasing the pump power or decreasing cavity loss at port 2) led to pulse breakup into two temporally separated pulses, as would be expected for this type of cavity [16]. All results reported here are below the threshold energy for such pulse breakup. The Yb-fiber single-pass gain was estimated to be $\sim 19.7 \mathrm{~dB}$, from the $0.5 \mathrm{~mW}$ power at the input to the fiber section to the $47.2 \mathrm{~mW}$ output power from the fiber. For completeness, we should add that the single-wavelength operation state more typically associated with this cavity type was also observed experimentally.

The spectra from ports 1 and 2 are shown in the inset in Fig. 2. The spectral bandwidth from port 1 is $32 \mathrm{~nm}$ (FWHM), and significant power is seen in the spectral wings, giving a $-10 \mathrm{~dB}$ width of $72 \mathrm{~nm}$. Port 2 outputs a modulated spectrum with a $\sim 15 \mathrm{~dB}$ local minimum at $\sim 1064 \mathrm{~nm}$ and two temporally offset parts, with the more energetic part having a transform-limited duration of $39 \mathrm{fs}$ and a central wavelength of $1045 \mathrm{~nm}$. To calculate the full spectrum output from the fiber, the spectra from ports 1 and 2 were added together with appropriate energy scaling. The resulting broad, modulationfree output is shown in Fig. 4, indicating that a single pulse at the fiber output was divided into the three distinct output wavelengths at ports 1 and 2 .

Considering the hard spectral filtering provided by the iris, a significant amount of spectral red-shifting occurs during propagation in the cavity fibers. This could be caused mainly by self-phase modulation (SPM). We note that $\mathrm{Yb}$-fibers become strongly absorbing for wavelengths below $1030 \mathrm{~nm}$ in aluminosilicate fibers such as those used here, and that this will be a strong contributing factor to the asymmetric spectral broadening, as this absorption is not present for longer wavelengths.

Figure 5 shows the time domain output characteristics from ports 1 and 2 after an external grating-based compressor (not

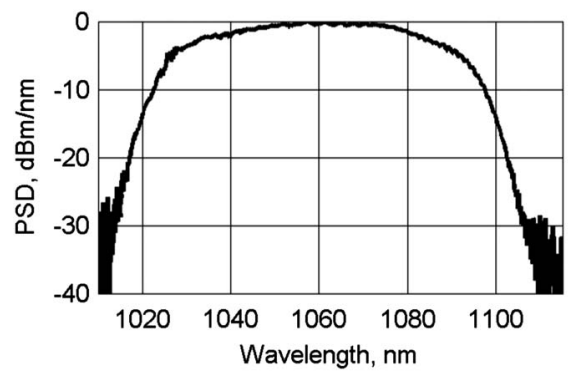

Fig. 4. Spectrum at the output of the fiber section before port 1, calculated by scaling the spectra from ports 1 and 2 and summing them (see individual spectra from ports 1 and 2 inset in Fig. 2).

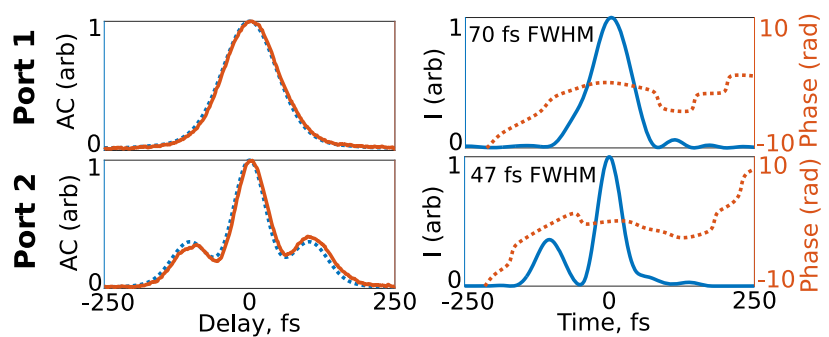

Fig. 5. Left column: measured (orange solid) and PICASOreconstructed (blue dotted) noncollinear second harmonic generation (SHG) autocorrelation data for ports 1 and 2 after an external grating based compressor. Right column: PICASO-reconstructed intensity (blue solid) and phase (orange dotted) for ports 1 and 2 .

shown in Fig. 2) had removed the chirp. The orange solid-line plots in the first column show the autocorrelations. These were used alongside the spectra from Fig. 2 with a Phase and Intensity from Cross-Correlation and Spectrum Only (PICASO) algorithm [23] to calculate the intensity and phase of the pulse, with results shown in the second column of Fig. 5. The PICASO-calculated autocorrelations are overlaid as dotted lines in the first column and are in close agreement with the experimental data. The spectrum from port 1 was used to calculate a transform-limited pulse duration of $47 \mathrm{fs}$ (intensity FWHM). The pulse from port 1 has a FWHM duration of $70 \mathrm{fs}$ (1.54 times transform limited, which is typical for this NPErejected port of this type of fiber laser). Some residual third-order dispersion remains, so the duration may be reduced further using a spatial light modulator (SLM)-based pulse shaper [24,25]. The PICASO calculations for port 2 show a primary pulse compressed to $47 \mathrm{fs}$. A secondary $63 \mathrm{fs}$ pulse, offset to longer wavelength, has a weak, nonzero and linearly changing phase, which may be due to a combination of the nonlinear effects of crossphase modulation (XPM) or SPM and the residual dispersion not compensated by the grating-compressor.

While it has been noted that for complex pulse shapes (e.g., highly chirped pulses) more advanced techniques such as X-FROG are valuable because PICASO pulse reconstructions can produce unreliable results, we have found during testing of the PICASO algorithm that it was robustly capable of reconstructing double-peaked pulses that are close to their transform-limited duration such as those presented here.

The extracavity compressor provided $-5.3 \times 10^{-4} \mathrm{fs}^{2}$ of second-order dispersion, whereas the intracavity compressor provided $-13 \times 10^{-4} \mathrm{fs}^{2}$. This means that the pulse was negatively chirped at the input to the cavity fibers, with residual negative spectral phase corresponding to $-7.7 \times 10^{-4} \mathrm{fs}^{2}$. The net dispersion of the cavity was low, and as such the pulse evolved following the standard dynamics for this stretchedpulse cavity design, reaching a minimum duration part way through the intracavity compressor and again part way through the cavity fibers. The negative chirp in the cavity fibers, along with the spectral filtering in the intracavity compressor, constrains the spectral broadening and allows for the self-consistent round-trip solution required for stable mode-locked operation, and contributes to both single- and multiwavelength operation. In detail, taking a typical value of $2.4 \times 10^{-4} \mathrm{fs}^{2} / \mathrm{m}$ for SMF 
and neglecting the nonlinear contribution to the spectral phase over the total $2.65 \mathrm{~m}$ length of passive fiber (permissible due to the low pulse energy in this section), a maximum fiber length of $3.2 \mathrm{~m}$ would be required to reach transform limit. This indicates that the minimum pulse duration (maximum intensity) is reached in the gain fiber after the pulse has undergone significant amplification, contributing to the overdriven NPE for this cavity.

The cavity remained stable for weeks, provided that there were no large changes in temperature to alter the cavity birefringence or any long term drifts in the alignment of the freespace cavity components. Qualitatively, the experimental cavity stability appeared to be slightly better in the multiwavelength state, but both single- and multiwavelength operations were reliable.

The RMS amplitude noise and timing jitter of the laser were characterized using the fundamental and 20th harmonic peaks of the RF spectrum alongside the method in Ref. [26], and are shown in Fig. 6. As no pedestal was seen for the fundamental RF peak, an upper bound for the noise amplitude was estimated to be $-80 \mathrm{~dB}$ (the noise floor for the measurement). The width of the noise was estimated using the width at the peak base ( $240 \mathrm{~Hz}$ with $10 \mathrm{~Hz}$ measurement resolution), giving an amplitude noise of $0.04 \%$. The timing jitter was estimated using the 20th harmonic of the RF spectrum, which had a peak-to-pedestal ratio of $45 \mathrm{~dB}$, giving a timing jitter of $4.12 \mathrm{ps}$ (width of the main peak: $30 \mathrm{~Hz}$; width of pedestal: $137 \mathrm{~Hz}$ ). The jitter characteristics of the multiwavelength mode-locking state therefore compares favorably with single wavelength oscillators of a similar design [4].

\section{SIMULATIONS}

\section{A. Model Development}

The overall goal of the simulations was to develop an understanding of the origin of the multiwavelength performance. While it was clear from the experimental observations of the laser stability and the RF spectrum that the behavior was not due to noise-like pulses and was also unlikely to be due to buildup of noise into a pulse created by stimulated Raman scattering (SRS), there remained a question about whether the pulses were highly coherent fragments of a single circulating
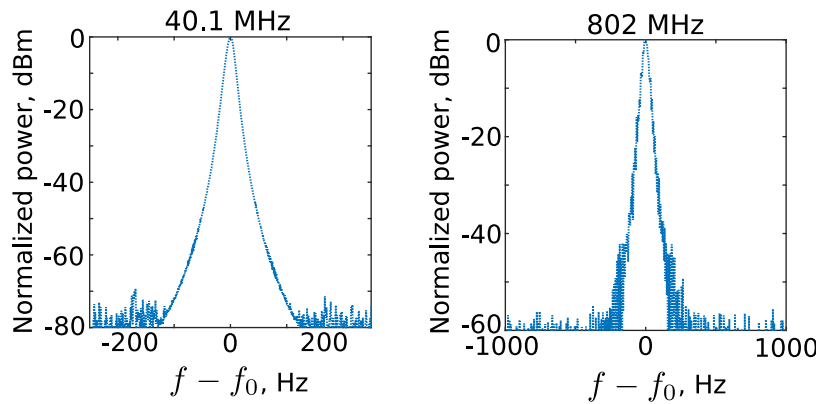

Fig. 6. RF spectral measurements taken from the oscillator in multiwavelength operation and used to calculate the RMS amplitude noise and timing jitter. Left: fundamental peak. Right: 20th harmonic. The resolution bandwidth was $10 \mathrm{~Hz}$. pulse or a bound pair of pulses with offset wavelengths but low mutual coherence.

The simulations used a simplified but still physically reasonable model for the gain and for the fiber birefringence, as this was not known in detail. The model used the experimental fiber lengths and included the vector nature of the polarization in order to highlight and understand the operation of the NPE switch. For the simulations, the Runge-Kutta Fourth-Order Interaction Picture (RK4IP) method [27] was used to numerically solve the generalized vector nonlinear Schrödinger equation. In particular, a variation formulated in the frequency domain was used with the Conservation Quantity Error Method (CQEM) [28] for adaptive step sizing, as this combination has been shown to reduce propagation error [27,29]. The field was represented by two $2^{12}$ arrays, one for both the $x$ - and $y$-polarization components of the pulse. The birefringence of the fiber was included by using a $\beta_{1}$ term in the dispersion profile of the fiber $y$-axis. The effects of chromatic dispersion, self-steepening, SPM, Raman scattering, spontaneous Raman noise [30,31], and XPM between orthogonally polarized field components were all included. The Jones matrix formalism was used to model the effects of the polarizationcontrolling optics on the amplitude and phase of the fields on the orthogonal axes.

The fiber GVD was set to $2.3 \times 10^{4} \mathrm{fs}^{2} / \mathrm{m}$ and the grating pair GDD was set to $-13 \times 10^{4} \mathrm{fs}^{2}$ to match the experimental value. Gain in the $\mathrm{Yb}$-fiber was included using a parabolic line shape and a gain per unit length of $G=2.9 \mathrm{~m}^{-1}$. The gain formula (shown in Eq. (1) below, where $|u|^{2}$ and $|\nu|^{2}$ give the signal power on the fiber $x$ - and $y$-polarization axes, respectively) included a saturation term with saturation fluence of $E_{\text {sat }}=0.8 \mathrm{~nJ}$, which is above the pulse energy we achieve here, so only modest gain shaping would occur. The simulated gain peak was set to $1060 \mathrm{~nm}$, and the gain bandwidth was set to $100 \mathrm{~nm}$. These values were found to give a good match between the simulated and experimental output powers. Minor effects caused by variations in the inversion along the fiber leading to variations in the gain spectrum in the experimental cavity have not been included, but this is unlikely to have a major effect on the conclusions drawn from the modeling. The group velocity mismatch (GVM) between the two polarization components was set to $-15 \mathrm{fs} / \mathrm{m}$ for the $y$-axis after following a trial-and-error approach and the repetition rate was set to match the experimental cavity $(R=40 \mathrm{MHz})$ :

$$
g=G \exp \left(-\frac{\int\left(|u|^{2}+|\nu|^{2}\right) \mathrm{d} t}{E_{\text {sat }}}\right) .
$$

Spectral filtering was included in the simulations to represent the bandpass filtering provided by the iris in the intracavity compressor. This was simulated by defining a square filter in the spectral domain and applied by multiplying with the pulse spectrum.

The model cavity demonstrated self-starting behavior from a starting condition of quantum noise (one photon per mode per polarization axis [30]), with a $25 \mathrm{fJ}, 1 \mathrm{~nm}$ bandwidth, unchirped Gaussian pulse added to reduce by an order of magnitude the number of round trips required for a mode-locked solution to stabilize. For simplicity, the central wavelength of the seed pulse was set to the $1060 \mathrm{~nm}$ gain peak. Much lower 
energy seeding normally enabled convergence to mode-locked solutions, but would occasionally lead to vector modulation instability, preventing stable operation over many round trips $[32,33]$.

\section{B. Simulation Results}

The buildup of mode locking from the starting conditions is shown for the single wavelength case in Fig. 7(a). The time and spectral domain data for port 1 is shown in the first two columns. The same is shown for port 2 in the third and fourth columns. The temporal and spectral pulse shapes are shown for the 200th round trip above each column. In this singlewavelength case, the pulses emitted from both ports of the simulated cavity are similar, each with a single peak in time domain and wavelength domain (centered at $\sim 1064 \mathrm{~nm}$ ). The change in position of the pulse in the time window with round trip number is attributed to the fact that the major axis of the polarization ellipse is oriented toward the faster $y$-axis at the input to the fiber section, which speeds up the field during the initial stages of mode locking. Once the mode-locked

\section{(a) Single wavelength}
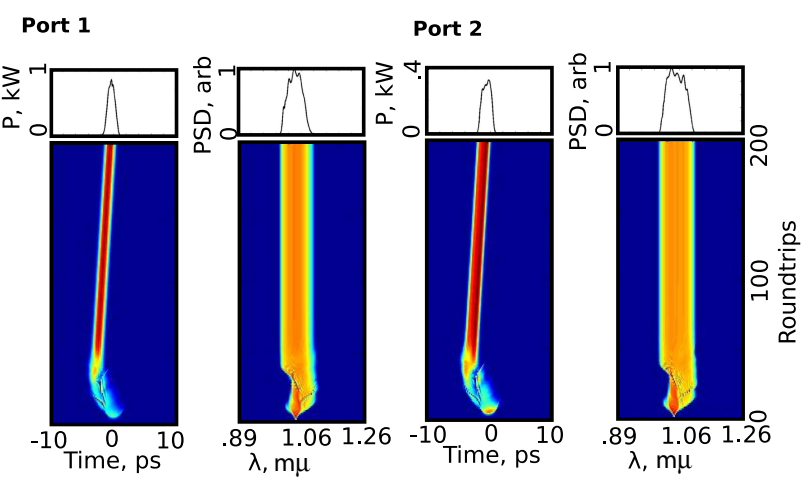

(b) Multiwavelength

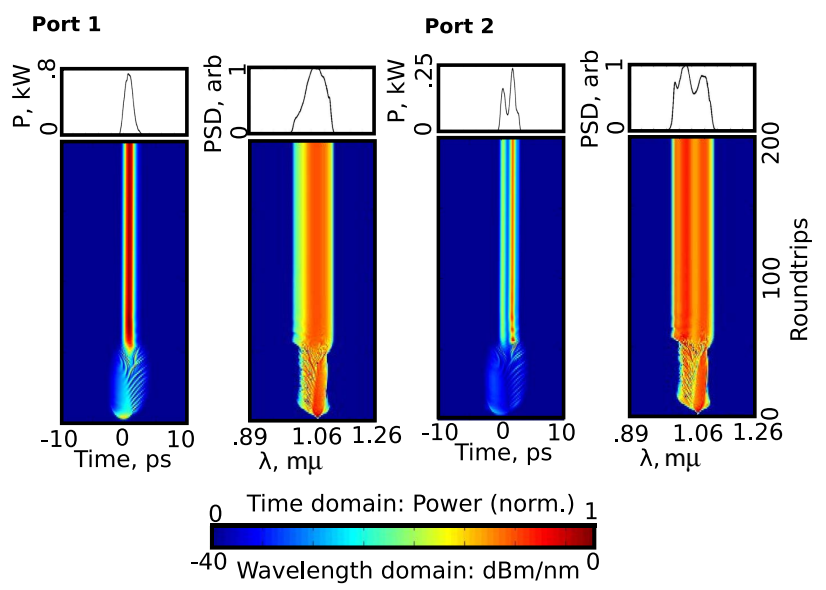

Fig. 7. Simulations showing mode locking initiated from quantum noise. (a) Single wavelength output state, and (b) multiwavelength output state. The first and second columns show the time domain (normalized power) and spectral domain $(\mathrm{dBm} / \mathrm{nm})$ results for port 1 , and the third and fourth columns show the same data for port 2. The corresponding color map shows both normalized instantaneous power and normalized spectral power density. state stabilizes, the power is distributed more toward the $x$-component of the field due to the NPE, and the pulse is delayed accordingly.

The multiwavelength results are shown in Fig. $7(\mathrm{~b})$ in the same format as for the single-wavelength case. The model reproduces the dynamics observed for the experimental cavity and the output from port 1 has a single peak in both time and wavelength domains, with a central wavelength of $1060 \mathrm{~nm}$. The pulse duration measured from this port in the simulated cavity was $2.6 \mathrm{ps}$, which is in reasonable agreement with the experimentally measured value of $\sim 3$ ps. The output from port 2 has a double peak, corresponding to the two pulses seen in the experimental data in Section 2. In contrast to the singlewavelength state, the pulse is centered in the time domain throughout the evolution to the mode-locked state because the polarization at the input to the fiber is exactly circular, with no power imbalance between the fast and the slow polarization axes.

The governing parameter selecting between single- and multiwavelength modes was the adjustment of the waveplate angles, and no other parameters were changed when moving from one state to the other. The half and QWP rotation/ retardances are shown in Table 1. For multiwavelength operation, it was important to have full circular polarization at the input to the fiber section in the simulations. The polarization state before the QWP at the input to the fiber section was linear and changing the QWP retardance by $20 \%$ from $\lambda / 4$ to $0.8 \times \lambda / 4$ was sufficient to switch from multiwavelength to single-wavelength operation. In multiwavelength operation, the $\mathrm{QWP}_{\mathrm{IN}}$ angle was within three degrees of the angle required for circular polarization at the input to the fibers in the experimental cavity, which is in good agreement with the observation from the simulations that circularly polarized input to the fiber was important for multiwavelength operation. The simulations confirm that the single-wavelength and multiwavelength mode-locking states are both stable, and that the pulses remain coherent over many round trips. The standard breathing pulse behavior was observed for all simulations performed, and spectral filtering acted to create larger bandwidths than would be possible in its absence because subsequent SPM and dispersion act on a shorter, narrower bandwidth pulse [21]. However, the simulation results shown in Fig. 7(b) show only $\mathrm{a} \sim 3 \mathrm{~dB}$ central minimum in the output from port 2 , whereas the experimental cavity shows a $>10 \mathrm{~dB}$ minimum. This is due to small differences in the model parameters in comparison with the experimental fibers. For example, in the simulations, the orientation of the fiber major and minor axes was not changed at each splice point.

Table 1. Polarization Rotation and Retardance for the Single and Multiwavelength Operation of the Vector Cavity Model

\begin{tabular}{lcc}
\hline Parameter & $\begin{array}{c}\text { Single Wavelength } \\
\text { Rotation/Retardance }\end{array}$ & $\begin{array}{c}\text { Multiwavelength } \\
\text { Rotation/Retardance }\end{array}$ \\
\hline $\mathrm{HWP}_{\mathrm{NPE}}$ & $0.91 \pi$ & $1.03 \pi$ \\
$\mathrm{QWP}_{\mathrm{NPE}}$ & $0.24 \times \lambda / 4$ & $-0.4 \times \lambda / 4$ \\
$\mathrm{QWP}_{\mathrm{IN}}$ & $0.8 \times \lambda / 4$ & $\lambda / 4$ \\
\hline
\end{tabular}


The wavelength to time mapping for the multiwavelength pulses from ports 1 and 2 are shown by the spectrograms in Fig. 8. The pulses are clearly emitted with a positive and predominantly linear chirp, which results in the straight line running through the density plots from bottom left to top right. Through this chirp map, the intensity-dependent polarization state is seen also to be a function of wavelength. The details of the NPE process are illustrated by the polarization schematics inset in the top left subplot. The polarization is elliptical at the extremes of the spectrum, with short wavelengths having counterclockwise elliptical polarization and long wavelengths having clockwise elliptical polarization. In contrast, the central part of the pulse is linearly polarized. The initially elliptically polarized parts of the pulse pass through the NPE switch to port 2 without significant attenuation, whereas the linearly polarized central part of the pulse is strongly ejected at port 1 , which has a positively chirped single spectral peak. The port 2 output is formed of two positively chirped pulses with distinct central wavelengths, as shown in the bottom right of Fig. 8.

The pulses generated in the single-wavelength simulations have a similar linear pulse chirp, but more homogeneous polarization state at the NPE switch. This is because XPM is reduced by the change in ellipticity at the input to the fiber section from the circular polarization required for multiwavelength operation. In effect, the unequal power on the two polarization axes weakens the NPE.

The evolution of the signal power calculated at the output of the fiber section of the cavity is shown in Fig. 9 for both singleand multiwavelength regimes. The power stabilized after $\sim 100$ round trips. The power is higher in the multiwavelength case and the calculated value of $50 \mathrm{~mW}$ is close to the $47.2 \mathrm{~mW}$ estimated at the same point in the experimental cavity. The higher peak power commensurate with the higher circulated
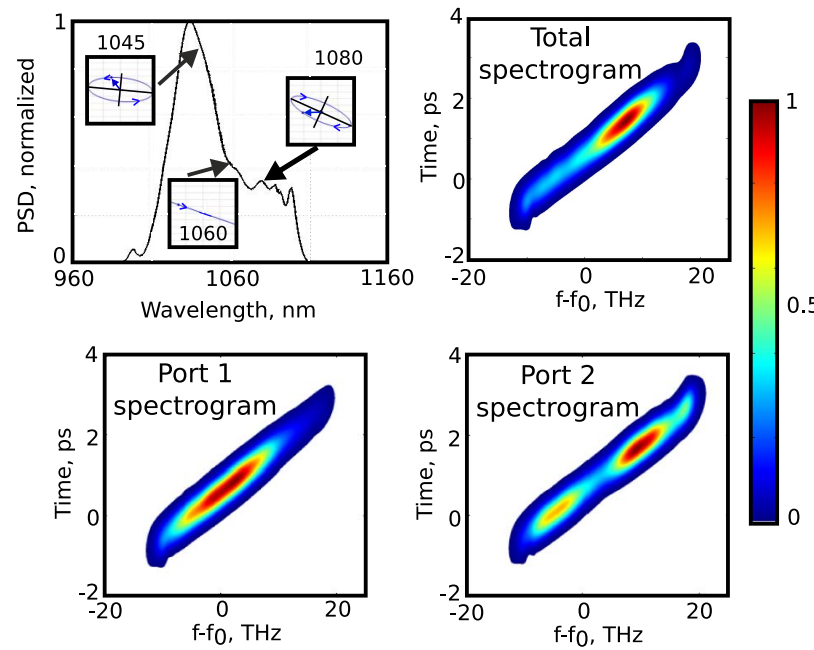

Fig. 8. Top left: simulated spectrum of the multiwavelength modelocked pulse just before port 1 for the 200th cavity round trip. Insets: polarization state at three different wavelengths (propagation axis directed out of the page). Top right: spectrogram of the same pulse on a linear scale, showing the wavelength to time mapping. Bottom left: spectrogram of the single pulse emitted from port 1. Bottom right: spectrogram of the double pulse emitted from port 2. The color map is normalized to the maximum value for each spectrogram. $\Delta f=42 \mathrm{GHz}, \Delta t=7 \mathrm{fs}$, and $f_{0}=283 \mathrm{THz}(1060 \mathrm{~nm})$.

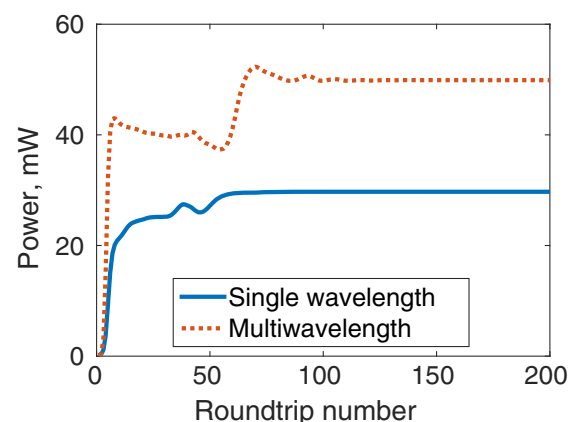

Fig. 9. Power evolution as a function of round-trip number for the simulated single- and multiwavelength cavities, measured directly after the cavity fibers.

average power for the multiwavelength state contributes to the overdrive of the NPE switch. The total output coupling is higher for single-wavelength operation, which resulted in the relatively lower circulated power.

Overall, the simulations show good qualitative agreement with the experimental data and represent, to our knowledge, the first report of such numerical results showing multiwavelength mode locking. Small differences between experimental and numerical results are inevitable, given that the simulations use an approximation for the $\mathrm{Yb}$ gain profile and fiber birefringence. The simulations clearly point out the mechanism underlying the observed experimental behavior.

\section{DISCUSSION AND CONCLUSION}

Previously, intracavity spectral filters have been used to form a multipeaked gain spectrum supporting more than one modelocked pulse in an Er-fiber laser [34]. This differs from the approach used here, as the gain itself is broad and smooth, but the pulse passes through a spectral filter each round trip that blocks the long wavelength part of the multipeaked output. Hence, the multiple wavelengths are due to SPM acting each round trip to broaden the spectrum, and then the action of nonlinear polarization evolution carves multiple wavelengths from that single chirped pulse at the polarization-based output switch. The single circulating pulse mechanism eliminates the effect of GVM between two independent pulses, and hence maintains a fixed temporal separation between the different wavelength pulses at the laser output. In contrast, pulse walk-off has been observed with other strategies for demonstrating multiwavelength cavities, e.g., Ref. [34].

The observation that the pulses are strongly chirped at the output port, which creates an approximately linear map between the time domain and the wavelength domain, is key to the multiwavelength operation of the laser. Simulations show that in multiwavelength operation, there is a strong variation of the polarization state across the chirped pulse, which is caused by an excess of XPM compared to single-wavelength operation. This leads to the formation of a nonlinear birefringent filter that separates the circulated pulse into subpulses with distinct central wavelengths. While this is in some ways similar to the case described in Ref. [35], where a Lyot filter based on purely linear components was used for bandwidth control in an 
all-normal dispersion cavity, the chirped output pulses from the laser reported here had a much larger bandwidth and were compressible to a duration of $47 \mathrm{fs}$ using an external grating compressor.

The experimental observations and the simulation results both support the suggestion that the mechanism creating the multiwavelength operation is an overdriven NPE switch acting on a chirped broad-bandwidth pulse (in the experiment, the pulse duration was $\sim 3$ ps at port 1 , with a $72 \mathrm{~nm}$ bandwidth at the $-10 \mathrm{~dB}$ level). Furthermore, in the experiments, the iterative method of mode locking the laser involved selecting the QWP $\mathrm{IN}_{\mathrm{IN}}$ angle (and hence retardance), then searching for a combination of $\mathrm{QWP}_{\mathrm{NPE}}$ and $\mathrm{HWP}_{\mathrm{NPE}}$ angles that produced mode-locked pulses. If no stable mode locking was achieved, then the angle of $\mathrm{QWP}_{\mathrm{IN}}$ was changed and the process repeated. For a given $\mathrm{QWP}_{\mathrm{IN}}$ setting, we observed either single- or multiwavelength operation, but not both, which corroborates the observation from the simulations that the $\mathrm{QWP}_{\mathrm{IN}}$ angle determined the possible mode of operation of the cavity, and not the QWP $\mathrm{NPE}_{\mathrm{NP}}$ and $\mathrm{HWP}_{\mathrm{NPE}}$ angles.

In the simulations of an idealized version of the cavity, it was possible to show directly that the NPE leads to a varying polarization state across the pulse and that this can lead to the NPE switch carving out a section of the spectrum and allowing longer and shorter wavelengths to circulate. The simulations showed that the QWP IN setting was the main factor controlling the degree of variation in polarization state across the pulse at the input to the NPE switch. The calculation of output power from the simulated cavity also showed higher power for multiwavelength operation. As with the experiments, the central $1064 \mathrm{~nm}$ wavelength band is output at the NPE switch (port 1) and a fraction of the power in each of the wings at 1045 and $1080 \mathrm{~nm}$ is output at port 2, so there are three distinct pulse wavelengths. Both experimental and simulated cavities showed this mode of operation is self-starting and stable. In addition, the results show how the mode-locked laser might become a suitable platform for studying the nonlinear dynamics underlying the bifurcation of the output pulse wavelength in the future.

In summary, the mechanism by which an excessive accumulation of nonlinear phase and a resulting overdrive of the NPE saturable absorption mechanism can create multiwavelength operation of a mode-locked $\mathrm{Yb}$-fiber breathing pulse ring laser was described. A chirped pulse with a strongly varying polarization state versus time is divided into subpulses using intracavity polarizing optics to produce the observed spectra. The multiwavelength pulses are up to 2 orders of magnitude shorter (and having correspondingly broader bandwidths) than other reports of mode-locked multioutput sources in the literature.

Funding. Türkiye Bilimsel ve Teknolojik Araştirma Kurumu (TÜBITAK) (113F319); European Research Council (ERC) (ERC-617521).

Acknowledgment. The authors would like to thank Dr. A. Heidt for helpful suggestions in the early stages of the model development. They also thank M. Zhang and Prof. D. Richardson for technical assistance. Data from the figures is available from the University of Southampton repository at [36].

\section{REFERENCES}

1. B. Oktem, C. Ulgudur, and F. Ö. Ilday, "Soliton-similariton fiber laser," Nat. Photonics 4, 307-311 (2010).

2. K. Tamura, E. P. Ippen, H. A. Haus, and L. E. Nelson, "77 fs pulse generation from a stretched-pulse mode-locked all-fiber ring laser," Opt. Lett. 18, 1080-1082 (1993).

3. R. Herda and O. G. Okhotnikov, "Dispersion compensation-free fiber laser mode-locked and stabilized by high-contrast saturable absorber mirror," IEEE J. Quantum Electron. 40, 893-899 (2004).

4. L. Lefort, J. H. V. Price, D. J. Richardson, G. J. Spühler, R. Paschotta, U. Keller, A. R. Fry, and J. Weston, "Practical low-noise stretchedpulse $\mathrm{Yb}^{3+}$-doped fiber laser," Opt. Lett. 27, 291-293 (2002).

5. H. Zhang, T. Dingyuan, R. J. Knize, L. Zhao, Q. Bao, and K. P. Loh, "Graphene mode locked, wavelength-tunable, dissipative soliton fiber laser," Appl. Phys. Lett. 96, 111112 (2010).

6. Z. Sun, D. Popa, T. Hasan, F. Torrisi, F. Wang, E. J. R. Kelleher, J. C. Travers, V. Nicolosi, and A. C. Ferrari, "A stable, wideband tunable, near transform-limited, graphene-mode-locked, ultrafast laser," Nano Res. 3, 653-660 (2010).

7. S. Wang, H. Yu, H. Zhang, A. Wang, M. Zhao, Y. Chen, L. Mei, and J. Wang, "Broadband few-layer $\mathrm{MoS}_{2}$ saturable absorbers," Adv. Mater. 26, 3538-3544 (2014).

8. D. Mao, S. Zhang, Y. Wang, X. Gan, W. Zhang, T. Mei, Y. Wang, Y. Wang, H. Zeng, and J. Zhao, "WS ${ }_{2}$ saturable absorber for dissipative soliton mode locking at 1.06 and $1.55 \mu \mathrm{m}$," Opt. Express 23, 2750927519 (2015).

9. D. Mao, B. Du, D. Yang, S. Zhang, Y. Wang, W. Zhang, X. She, H. Cheng, H. Zeng, and J. Zhao, "Nonlinear saturable absorption of liquid-exfoliated molybdenum/tungsten ditelluride nanosheets," Small 12, 1489-1497 (2016).

10. X. Zhao, Z. Zheng, L. Liu, Y. Liu, Y. Jiang, X. Yang, and J. Zhu, "Switchable, dual-wavelength passively mode-locked ultrafast fiber laser based on a single-wall carbon nanotube modelocker and intracavity loss tuning," Opt. Express 19, 1168-1173 (2011).

11. G. Sobon, J. Sotor, and K. M. Abramski, "All-polarization maintaining femtosecond Er-doped fiber laser mode-locked by graphene saturable absorber," Laser Phys. Lett. 9, 581-586 (2012).

12. Z.-C. Luo, A.-P. Luo, W.-C. Xu, H.-S. Yin, J.-R. Liu, Q. Ye, and Z.-J. Fang, "Tunable multiwavelength passively mode-locked fiber ring laser using intracavity birefringence comb filter," IEEE Photon. J. 2, 570-577 (2010).

13. S. Li and K. T. Chan, "A novel configuration for multiwavelength actively mode-locked fiber lasers using cascaded fiber Bragg gratings," IEEE Photon. Technol. Lett. 11, 179-181 (1999).

14. L. Yun, X. Liu, and D. Mao, "Observation of dual-wavelength dissipative solitons in a figure-eight erbium-doped fiber laser," Opt. Express 20, 20992-20997 (2012).

15. F. Ö. Ilday and F. W. Wise, "Multiple stable points of a soliton fiber laser with nonlinear polarization evolution," in Nonlinear Guided Waves and Their Applications 2001, OSA Technical Digest Series (Optical Society of America, 2001), paper MC80.

16. X. Liu, "Hysteresis phenomena and multipulse formation of a dissipative system in a passively mode-locked fiber laser," Phys. Rev. A 81, 023811 (2010).

17. V. J. Matsas, W. H. Loh, and D. J. Richardson, "Self-starting, passively mode-locked Fabry-Perot fiber soliton laser using nonlinear polarisation evolution," IEEE Photon. Technol. Lett. 5, 492-494 (1993).

18. F. Ö. Ilday, "Laser physics: turbulent times," Nat. Photonics 7, 767-769 (2013).

19. J. A. Cox, A. H. Nejadmalayeri, J. Kim, and F. X. Kärtner, "Complete characterization of quantum-limited timing jitter in passively modelocked fiber lasers," Opt. Lett. 35, 3522-3524 (2010).

20. F. Ö. Ilday, J. R. Buckley, H. Lim, F. W. Wise, and W. G. Clark, "Generation of 50-fs, 5-nJ pulses at $1.03 \mu \mathrm{m}$ from a wave-breakingfree fiber laser," Opt. Lett. 28, 1365-1367 (2003). 
21. P. Adel and C. Fallnich, "High-power ultra-broadband mode-locked $\mathrm{Yb}^{3+}$-fiber laser with $118 \mathrm{~nm}$ bandwidth," Opt. Express 10, 622-627 (2002).

22. G. P. Agrawal, Nonlinear Fiber Optics (Academic, 1989).

23. J. W. Nicholson, J. Jaspara, W. Rudolph, F. G. Omenetto, and A. J. Taylor, "Full-field characterization of femtosecond pulses by spectrum and cross-correlation measurements," Opt. Lett. 24, 1774-1776 (1999).

24. F. He, H. S. S. Hung, J. H. V. Price, N. K. Daga, N. Naz, J. Prawiharjo, D. C. Hanna, D. P. Shepherd, D. J. Richardson, J. W. Dawson, C. W. Siders, and C. P. J. Barty, "High energy femtosecond fiber chirped pulse amplification system with adaptive phase control," Opt. Express 16, 5812-5813 (2008).

25. J. Prawiharjo, N. K. Daga, R. Geng, J. H. V. Price, D. C. Hanna, D. J. Richardson, and D. P. Shepherd, "High fidelity femtosecond pulses from an ultrafast fiber laser system via adaptive amplitude and phase pre-shaping," Opt. Express 16, 15074-15089 (2008).

26. D. von der Linde, "Characterization of the noise in continuously modelocked lasers," Appl. Phys. B 39, 201-217 (1986).

27. J. Hult, "A fourth-order Runge-Kutta in the interaction picture method for simulating supercontinuum generation in optical fibers," J. Lightwave Technol. 25, 3770-3775 (2007).
28. A. Heidt, "Efficient adaptive step size method for the simulation of supercontinuum generation in optical fibers," J. Lightwave Technol. 27, 3984-3991 (2009).

29. A. A. Rieznik, A. M. Heidt, P. G. König, V. A. Bettachini, and D. F. Grosz, "Optimum integration procedures for supercontinuum simulation," IEEE Photon. J. 4, 552-560 (2012).

30. J. M. Dudley, G. Genty, and S. Coen, "Supercontinuum generation in photonic crystal fiber," Rev. Mod. Phys. 78, 1135-1184 (2006).

31. P. D. Drummond and J. F. Corney, "Quantum noise in optical fibers. 1. Stochastic equations," J. Opt. Soc. Am. B 18, 139-152 (2001).

32. G. P. Agrawal, "Modulation instability induced by cross-phase modulation," Phys. Rev. Lett. 59, 880-883 (1987).

33. G. P. Agrawal, P. L. Baldeck, and R. R. Alfano, "Modulation instability induced by cross-phase modulation in optical fibers," Phys. Rev. A 39 , 3406-3413 (1989).

34. D. Mao and H. Lu, "Formation and evolution of passively mode-locked fiber soliton lasers operating in a dual-wavelength regime," J. Opt. Soc. Am. B 29, 2819-2826 (2012).

35. K. Özgören and F. Ö. Ilday, "All-fiber all-normal dispersion laser with a fiber-based Lyot filter," Opt. Lett. 35, 1926-1928 (2010).

36. http://dx.doi.org/10.5258/SOTON/385536. 